\title{
Direct Manipulation of Web Browsing History
}

\author{
Gholam H. Khaksari \\ School of Information Sciences and Technology \\ Pennsylvania State University, Abington College \\ ghk10@psu. edu
}

\begin{abstract}
Working with Web browsing history provided by current browsers is a daunting task for Web users and a major source of user frustration. Users interact with Web browsing history using the Forward and Back navigation buttons and the bookmarks pull-down menus. This research presents an appropriate model for representation of Web browsing history with its related intuitive user interactions. Web browsing history is organized in a grid of thumbnail images including a set of manipulative control buttons with feedback. Through intuitive organization and direct manipulation of thumbnail images, users can achieve an enjoyable and productive Web browsing experience. As compared to using browser history through pull-down menus, Web users can rapidly switch their attention and move fluidly between different pages in the browsing history set without getting distracted and frustrated.
\end{abstract}

Keywords - Web Browsing History, Direct Manipulation, User Experience, Interaction Design, User Centred Design Introduction.

\section{INTRODUCTION}

If simplicity was a design requirement, then designers of the Web have clearly succeeded in creating the simplest global digital library in the history of mankind [1]. A user's mental model for the Web design can be described as: a set of hyperlinked documents, each identified by a unique address or Universal Resource Locator (URL).

But, was simplicity of use also a user-experience requirement?

A user fetches documents from the Web digital library by specifying a document URL in a browser address bar, or via conversing with a search engine using some search terms. However, as simple as it seems and despite all the fancy features provided by modern Web browsers, using the Web is not very simple for many users. One major source of Web user frustration is related to the Web browsing history features provided by current browsers. Generally speaking, a user revisits browsing history either sequentially via the Forward and Back navigation controls or by selection of a URL from the bookmarks (favorites) menus.

The goal of finding contextually relevant information on the Web always involves, among other things, the task of revisiting previously viewed pages. Use of the browser history features provided by modern browsers is very distracting and is not ideal. It can be a daunting cognitive task for many persons, above all for senior web users and those with cognitive limitations.

Microsoft Internet Explorer 8 saves a thumbnail image for the current Web page displayed under each browser tab in a page called Quick Tabs [2]. This page is accessed by a left mouse click on the Quick Tabs button located on the browser tabs bar. The Quick Tabs page provides for two very simple user actions. First, one for deleting a thumbnail image, which in effect 
International Journal of Computer Science \& Information Technology (IJCSIT) Vol 3, No 5, Oct 2011

deletes its corresponding tab; second, one that allows the user to return back to the original Web page associated with the thumbnail image. The Quick Tabs does not in any way alleviate user frustration related to Web browsing history; instead, it allows users to manage and easily switch between browser tabs.

Google Chrome 8 browser also has a New Tab page feature designed to help users to get to previously visited websites as quickly as possible [3]. Whenever the user opens a new tab, the New Tab page is preloaded with thumbnails of the websites user has visited the most. A left mouse click action on a thumbnail will take the user back to the corresponding website.

Apple Safari 5 Web browsing history features include the typical bookmarks menu and a slick Top Sites page [4]. Safari identifies favorite sites for placement on the Top Sites page based on how often and how recently they have been visited. To manually add a website to the Top Sites page is a bit awkward and not well designed for easy or effective use, which can be a source of user distraction. Customizing or editing the Top Sites page includes a reversible pin down action control to specify that a page should be kept in Top Sites and not replaced by other pages visited. To specify that a page should never be included in Top Sites, the action icon X located on the upper-left corner of the page thumbnail is clicked. Moreover, Safari provides a very nice drag and move feature to rearrange thumbnails on the Top Sites page.

Many attempts to improve the accessibility and management of Web browser history have been made, all lacking positive user experience focus. Instead, a new user-centered view of Web browsing is required to create a simple and enjoyable user experience. New forms of interactions with the Web such as direct manipulation and exploration through direct manipulation are required. An appropriate model for representation of Web browsing history with its related intuitive user interactions are required to provide a positive and productive user experience.

A better model of reality or an appropriate representation of Web browsing history would be a simple grid (as shown in Fig. 1) populated with thumbnail images of pages viewed by the user. The simple grid model of Web browsing history enables users to directly manipulate browser history data by simple mouse click interactions. This includes selecting and moving images vertically within columns, moving them under different columns, or deleting them from the history $[6,7,14,15,16,17]$. Through intuitive organization and direct manipulation of browsing thumbnail images all users can achieve a new Web browsing experience. As compared to using browsing history through pull-down menus, Web users can rapidly switch their attention and move fluidly between different pages in the browsing set without getting distracted and frustrated.

The model for representation of Web browsing history and its related intuitive user interactions must include design features that distinguish it from traditional solutions:

- Objects of interest are to be continuously represented on the grid

- Visible manipulative action buttons must be used instead of pull-down menu selections

- Actions must be rapid and reversible

- Visible feedback must be provided for every user action 


\section{II.CONCEPTUAL MODEL DESCRIPTION}

Let Web browsing history be the set of all Web pages that have been viewed and then captured for future reference by a Web user. An appropriate model for representation of the Web browsing history set is a grid populated with thumbnail images of the captured pages. Alternatively, Web page names may be used instead of thumbnail images. The Web browsing history grid consists of a number of labeled tabs or columns. Each tab in the grid corresponds one-to-one to its related tab found in the browser tab bar.

For example, the thumbnail images for the pages viewed and captured under the browser tab www.psu.edu are placed under the grid tab www.psu.edu of the browsing history grid. Under each grid tab one finds a set of thumbnail images, each image being the miniature visual view of a page viewed and captured by a user under its related browser tab. Thumbnail images under each grid tab are listed in chronological order with the most recent one at the top of each column.

The Web browsing history grid resides in the background, therefore it is not visible to users by default. A simple mouse click action on the Historian Button located on the browser tabs bar will display the grid history. The current browser display area is blurred out and replaced with the browsing history grid. Also, a simple mouse click action on the Historian Button will hide the browsing history grid. It is blurred out and replaced with the normal browser display. The user can easily toggle back and forth between browser view and browsing history grid via simple mouse click action on the Historian Button.

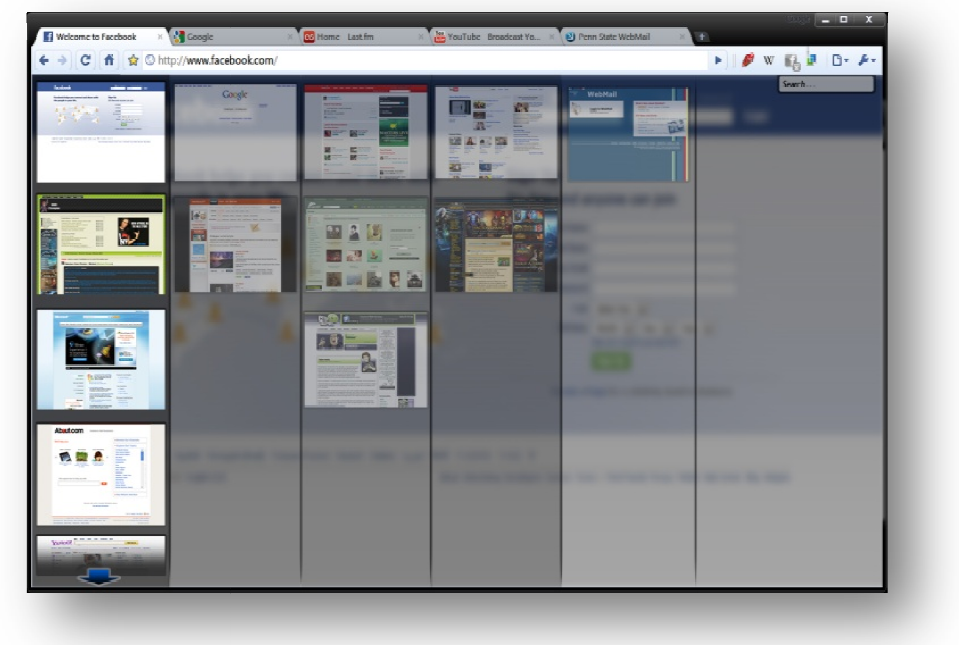

Fig. 1 Web Browsing History Grid

\section{ACTION BUTTON DESCRIPTION}

In addition to continuous representation of Web browsing history pages on the history grid, a set of rapid, reversible and incremental action buttons with feedback are provided. These action buttons allow a user to directly manipulate Web browsing history pages presented on the history grid. The action buttons are located on the browser tab bar, which are presented next. 
International Journal of Computer Science \& Information Technology (IJCSIT) Vol 3, No 5, Oct 2011

Also, each Web page Thumbnail may be moved up and down its own column or moved to any location under a different column using a left mouse click and hold and then move actions

\section{INTERACTING WITH CONCEPTUAL MODEL}

Typically, a Web user creates multiple browser tabs to conduct research on the Web known as tabbed browsing. The tabbed browsing feature enables a user to search multiple websites in a single browser window. For example, a user may create a tab for searching http://interactions.acm.org, a second tab for searching www.ieeeXplore.iee.org, and a third tab for searching www.google.com websites. A user can easily switch between browser tabs to conduct searches and view pages. When a user sees a webpage of interest, she can click on the Save Thumbnail Button located on the browser tab bar to store its thumbnail image on the history grid. The image is placed under a history grid tab corresponding to the browser tab where the web page is currently being viewed.

\begin{tabular}{|c|c|c|}
\hline Button & $\begin{array}{l}\text { Button } \\
\text { Name }\end{array}$ & Button Action Description \\
\hline & $\begin{array}{c}\text { Historian } \\
\text { Button }\end{array}$ & $\begin{array}{l}\text { Used to toggle back and forth between regular browser view } \\
\text { and browsing history grid view. User can easily toggle back } \\
\text { and forth between browser view and browsing history grid } \\
\text { view via simple mouse click action on the Historian Button. }\end{array}$ \\
\hline & $\begin{array}{l}\text { Grid } \\
\text { Display } \\
\text { Style } \\
\text { Button }\end{array}$ & $\begin{array}{l}\text { Used to toggle back and forth between the thumbnail image } \\
\text { view style and the list view style of browsing history grid. } \\
\text { Saved Web pages may be represented on the history grid by a } \\
\text { thumbnail image or the Web page URL. }\end{array}$ \\
\hline & $\begin{array}{l}\text { Save } \\
\text { Thumbnail } \\
\text { Button }\end{array}$ & $\begin{array}{l}\text { Used for saving a thumbnail image of the current Web page } \\
\text { being viewed by user and the subsequent placement of the } \\
\text { thumbnail image on the history grid. The thumbnail image is } \\
\text { placed under a history grid tab corresponding to the browser } \\
\text { tab where the web page is being viewed. }\end{array}$ \\
\hline & $\begin{array}{l}\text { Cut } \\
\text { Thumbnail } \\
\text { Button }\end{array}$ & $\begin{array}{l}\text { Used to cut a selected thumbnail image from the browsing } \\
\text { history grid. The thumbnail image must be selected before it } \\
\text { can be cut. Alternatively, user may click on the delete marker } \\
\text { (X) located on the upper-right corner of the thumbnail image to } \\
\text { delete it. }\end{array}$ \\
\hline & $\begin{array}{l}\text { Undo Last } \\
\text { Action } \\
\text { Button }\end{array}$ & $\begin{array}{l}\text { Used to undo the last user action. The Web browsing history } \\
\text { grid will be restored to its prior state before the last action } \\
\text { taken by the user. }\end{array}$ \\
\hline
\end{tabular}


International Journal of Computer Science \& Information Technology (IJCSIT) Vol 3, No 5, Oct 2011

\begin{tabular}{|c|c|c|}
\hline 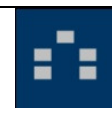 & $\begin{array}{l}\text { Scroll-up } \\
\text { Button }\end{array}$ & $\begin{array}{l}\text { Used to scroll up thumbnail image column under active } \\
\text { browsing history grid tab. }\end{array}$ \\
\hline & $\begin{array}{l}\text { Scroll- } \\
\text { down } \\
\text { Button }\end{array}$ & $\begin{array}{l}\text { Used to scroll down thumbnail image column under active } \\
\text { browsing history grid tab }\end{array}$ \\
\hline & $\begin{array}{c}\text { Copy } \\
\text { Thumbnail } \\
\text { Button }\end{array}$ & $\begin{array}{l}\text { Used to copy a selected thumbnail image from the browsing } \\
\text { history grid. The thumbnail image must be selected before it } \\
\text { can be copied. }\end{array}$ \\
\hline & \begin{tabular}{c|} 
Paste \\
Thumbnail \\
Button
\end{tabular} & $\begin{array}{l}\text { Used to paste a copied thumbnail image in the location pointed } \\
\text { to by mouse on the browsing history grid. }\end{array}$ \\
\hline
\end{tabular}

Fig. 2 Web Browsing History Action Button

A Web user can easily toggle back and forth between browser view and browsing history grid view via simple mouse click action on the Historian button. Once in the browser history grid, a user moves the mouse cursor over the browsing grid to look at thumbnail images as a librarian uses a microscope to examine a matrix of archived microfilm images. When the mouse cursor is moved over grid tabs, the tab located directly under the cursor and its thumbnail images will appear in the forefront. The tab directly under the cursor can become active by a simple mouse left click action. A user moves the mouse cursor vertically over the thumbnail images under the active tab to magnify them for closer examination. These activities (as shown in Fig. 2) are support:

- Activating Browsing History Grid: User activates the browsing history grid by pointing mouse cursor to the Historian Button followed by a left-click action. Once activated, the current browser display area is blurred out and replaced with browsing history grid.

- Activating Browser Display: User toggles back to browser display by pointing the mouse cursor to the Historian Button followed by a left-click mouse. Once activated, browsing history grid area is blurred out and replaced with browser display.

- Toggling Browsing History Grid Style: User toggles back and forth between two distinct browsing history grid styles by pointing the mouse cursor to the Grid Display Style Button followed by a left-click action.

- Saving Thumbnail Images: User saves the thumbnail image for the displayed Web page by pointing the mouse cursor to Save Thumbnail Button followed by a left-click action. The thumbnail image of the current page is placed under browsing history grid tab with same name as the current browser tab. 
International Journal of Computer Science \& Information Technology (IJCSIT) Vol 3, No 5, Oct 2011

- Deleting Thumbnail Image: User deletes a thumbnail image from browser history grid by pointing the mouse cursor to the thumbnail image followed by a left-click action to select it, and a left-click action on the Cut Thumbnail Button.

- Undoing Last Interaction: User can undo their last action by pointing the mouse cursor to the Undo Last Action Button followed by a left-click action.

- Manipulating Thumbnail Images: User selects a thumbnail image by pointing mouse cursor to an image on the grid followed by a left-click-hold action. Once selected, the image can be moved vertically within its own tab or moved under a different tab.

- Copying a Thumbnail Image: User copies a selected thumbnail image from the browsing history grid. The thumbnail image must be selected before it can be copied.

- Pasting a Thumbnail Image: User pastes a copied thumbnail image in the location pointed to by the mouse on the browsing history grid.

- Scrolling-up Browsing Images: User can scroll up the browser history grid image using the browser scroll-up controls.

- Scrolling-down Browsing Images: User can scroll down the browser history grid image using the browser scroll-down controls.

- Using the Search Bar: User can type search terms in the provided search bar to search for objects in the browsing history.

\section{V.CONCLUSION}

The proposed model organizes Web browsing history pages on a grid of tabs populated with thumbnail images of pages viewed and saved by the user. Furthermore, it provides a set of manipulative control buttons with feedback for direct manipulation of the model. Simplicity of use for all users has been an important design concern. The model provides at least these values:

- Reduced Cognitive Workload: Interactions between the user and the model are through direct manipulation of the thumbnail images as opposed to menu interactions reducing user cognitive workload.

- Minimal User Distraction and Frustration: As compared to using traditional browser history through pull-down menus, Web users can rapidly switch their attention and move fluidly between different pages in the browsing history set without getting distracted or frustrated.

- Enjoyable Web Browsing Experience: Through intuitive organization and direct manipulation of thumbnail images, users can achieve an enjoyable Web browsing experience.

- New Content Aggregation Method: Using the very simple grid of tabs design and related manipulative controls, knowledge workers can conduct Web research and aggregate content from multiple sources. 
International Journal of Computer Science \& Information Technology (IJCSIT) Vol 3, No 5, Oct 2011

- New User-Centered Focus: The proposed model provides a new user-centered focus for using the Web. Many attempts to improve the accessibility and management of Web content have been made, all lacking positive user experience focus.

\section{ACKNOWLEDGMENT}

The author would like to thank all Penn State University- Abington IST students specially Adam Trinh for their enthusiastic interest and contributions to the field of Human Computer Interaction.

\section{RELATED WORK}

A large sum of research effort related to accessibility and management of browser page-view history lack consideration for users with cognitive disabilities $[8,9,10,11,12,13]$. Several ideas for supporting page-view re-visitation are discussed in [8]. Authors in [9] propose a GoalTask model to organize and retrieve browsing histories by associating purposes (goal) with works (task) on Web browsing. Several possible types of interactions that users could have with document histories data are presented in [10]. Paper [11] compares web navigation histories between two users.

\section{About the Author}

Gholam H. Khaksari, D.Sc. is a 25-year veteran of the software development industry before joining academia. He serves on the faculty of the School of IST at Pennsylvania State University- Abington College. When teaching his students, he draws on his real world experience of programming languages and software development. He earned his Doctor of Science degree in Applied Information Technology (AIT) at Towson University, and his Masters in Computer Science at Johns Hopkins University. His research interests include HCI, VoIP, Security, and Expert Systems. He completed his dissertation on the design, implementation, and performance of a novel VoIP softphone that runs on a bare IA-32 based PC without an operating system, an alternative approach that provides secure, reliable and efficient voice communication.

\section{REFERENCES}

[1] "Berners-Lee, Tim; Cailliau, Robert (November 12, 1990). "World Wide Web: Proposal for a hypertexts Project."http://w3.org/Proposal.html. Retrieved July 27, 2009.

[2] Microsoft Internet Explorer 8; http://www.microsoft.com/windows/internet-explorer/ie7/

[3] Google Chrome 8; http://www.google.com/chrome/

[4] Apple Safari 5; http://www.apple.com/safari/

[5] Mozilla Firefox; http://www.mozilla.com/

[6] Shneiderman, Ben and Plaisant, Catherine (2005): Designing the User Interface: Strategies for Effective Human-Computer Interaction (4th ed.). Addison-Wesley

[7] Sharp, Helen, Rogers, Yvonne and Preece, Jennifer J. (2007): Interaction Design: Beyond HumanComputer Interaction. John Wiley and Sons

[8] Steve Won, Jing Jin, and Jason I. Hong. Contextual web history: using visual and contextual cues to improve web browser history. In Proceedings of the 27th international conference on Human factors in computing systems (CHI '09) April 2009 
International Journal of Computer Science \& Information Technology (IJCSIT) Vol 3, No 5, Oct 2011

[9] Kenji Higuchi, Fumiko Harada, and Hiromitsu Shimakawa: Making links between goal and tasks to retrieve information from web browsing histories. In proceedings of the 2nd International Conference on Interaction Sciences: Information Technology, Culture and Human (ICIS '09) November 2009.

[10] Adam Jatowt, Yukiko Kawai, Hiroaki Ohshima, an dKatsumi Tanaka: What can history tell us?: towards different models of interaction with document histories. In Proceedings of the nineteenth ACM conference on Hypertext and hypermedia (HT '08) June 2008.

[11] Mark Bilezikjian, John C. Tang, James "Bo" Begole, and Nicole Yankelovich: Exploring web browser history comparisons. In preceding of $\mathrm{CHI}$ '02 extended abstracts on Human factors in computing systems (CHI '02) April 2002

[12] Abrams, D., R. Baecker, and M. Chignell: Information archiving with bookmarks: personal webspace construction and organization. In Proceedings of CHI'98: ACM Press.

[13] Adar, E., J. Teevan, and S. Dumais: Large Scale Analysis of Web Revisitation Patterns. In Proceedings of Twenty-sixth annual SIGCHI conference on Human factors in computing systems (CHI 2008).

[14] Miguel Carvalhais: Learning and studying interaction design through design patterns. In proceedings of the 15th Conference on Pattern Languages of Programs (PLoP '08) October 2008

[15] Carroll, John M. (ed.) (2003): HCI Models, Theories, and Frameworks: Toward a multidisciplinary science. San Francisco, Morgan Kaufmann Publishers

[16] Transactions. http://interactions.acm.org/

[17] W3C Web Accessibility Initiative. http://www.w3.org/WAI/ 